\title{
VERSAL UNFOLDINGS OF $G$-INVARIANT FUNCTIONS
}

\author{
BY V. POÉNARU
}

Communicated May 6, 1975

1. We announce here some results on equivariant local differential analysis. The proofs will appear elsewhere [7]. We consider a compact Lie group $G$, acting orthogonally on $R^{n} \cdot C^{\infty}(x)$ (respectively $C^{\infty}\left(R^{n}\right)$ ) will denote the ring of germs of $C^{\infty}$ functions around $0 \in R^{n}$ (the ring of $C^{\infty}$ functions of $\left.R^{n}\right)$. The germ of $R^{n}$ at 0 will be denoted by $X . C^{\infty}(x)^{G}, C^{\infty}\left(R^{n}\right)^{G}$ will denote the $G$-invariant germs (functions). We shall consider parameter (germs of) spaces $U, V, \ldots$, on which $G$ acts, by definition, trivially.

If $f(x) \in C^{\infty}(x)^{G}$, an unfolding of $f(x)$ is an $F(x, u) \in C^{\infty}(x, u)^{G}$ such that $F(x, 0) \equiv f(x)$. The unfolding $F(x, u)$ is versal, if any other unfolding of $f(x), H(x, v) \in C^{\infty}(x, v)^{G}$, can be induced from $F$, by a commutative diagram

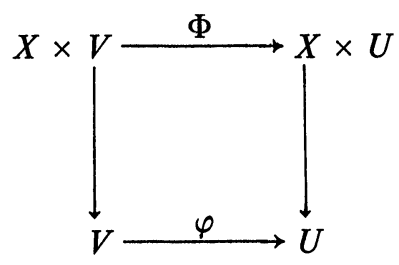

such that:

(a) $\Phi, \varphi \in C^{\infty}$,

(b) $\Phi$ is $G$-equivariant,

(c) $\Phi \mid X \times 0 \equiv$ id $X$,

(d) $H=F \circ \Phi$.

$G$ also acts on smooth vector-fields on $X\left(R^{n}\right)$. We consider the invariant (germs of) vector-fields $\Gamma^{\infty}(T X)^{G} \subset \Gamma^{\infty}(T X)$ i.e., fields such that $g \xi(x)=$ $\operatorname{Tg}(\xi(x))=\xi(g x) . \Gamma^{\infty}(T X)^{G}$ is a $C^{\infty}(x)^{G}$-module moreover, if $f(x) \in C^{\infty}(x)^{G}$, the subset

$$
J_{G}(f)=\left\{d f(\xi), \xi \in \Gamma^{\infty}(T X)^{G}\right\} \subset C^{\infty}(x)^{G} .
$$

is an ideal, called the G-jacobian ideal of $f$. We shall assume that $f$ is given, and that $\operatorname{dim}_{R} C^{\infty}(x)^{G} / J_{G}(f)<\infty$.

By definition $F(x, u) \in C^{\infty}(x, u)^{G}$, unfolding of $f$, is infinitesimally versal if the images of $\partial F(x, 0) / \partial u_{1}, \ldots, \partial F(x, 0) / \partial u_{k}$ in $C^{\infty}(x)^{G} / J_{G}(f)$ generate the $R$-vector space $C^{\infty}(x)^{G} / J_{G}(f)$.

AMS (MOS) subject classifications (1970). Primary $58 \mathrm{C25}$. 
THEOREM 1. If the unfolding $F(x, u) \in C^{\infty}(x, u)^{G}$ (of $f(x) \in C^{\infty}(x)^{G}$ ) is infinitesimally versal, it is versal.

This is a generalization of a result of J. Mather [5], R. Thom [16], V. M. Zakalyukin [14], F. Sergeraert [10], G. Lassalle [3], and others.

This theorem should be useful for "catastrophy theory in the presence of symmetry" [11], [12].

2. The main ingredient for proving Theorem 1 is the equivariant preparation theorem, which we describe now.

Suppose $G$ (compact Lie group) acts orthogonally on $R^{n}, R^{p}$; the germs of these two spaces, around 0 , will be denoted by $X, Y$.

We consider a germ of smooth map $f \in C^{\infty}(X, Y)$ which is equivariant: $f(g x)=g f(x)$. Then $f$ induces a local ring homomorphism $C^{\infty}(x)^{G} \stackrel{f^{*}}{\longleftarrow}$ $C^{\infty}(y)^{G}$.

THEOREM 2. If $M$ is a finitely generated $C^{\infty}(x)^{G}$-module, such that $\operatorname{dim}_{R} M / f^{*} M C^{\infty}(y)^{G} \cdot M<\infty$, then $M$ is also finitely generated as a $C^{\infty}(y)^{G}$. module.

This is a generalization of a theorem of B. Malgrange [4] and J. Mather [6].

3. This paragraph provides some examples for Theorem 1.

With $G$ compact as before we consider the algebra of $G$-invariant polynomials $R[x]^{G}$. By a classical result of Hilbert [2], [13], this algebra is finitely generated, i.e. there is a polynomial map $y=\rho(x)\left(R^{n} \stackrel{\rho}{\longrightarrow} R^{p}\right)$ (given by finitely many homogenous polynomials, of positive degree), such that $R[x]^{G}$ $\stackrel{\rho^{*}}{\longleftarrow} R[y]$ is surjective. It had been conjectured, for some time, that this is still true in the $C^{\infty}$ case. In fact G. Glaeser [15] had proved it for $G=$ the symmetric group, and for some time at least the local case for finite $G$ has been known to result from the preparation theorem (see for example [1]). Note also that there is a way to work along the diagonals and go from the local to the global case. Now, the general compact case has been proved by G. Schwarz [9], and it is this result which makes the present paper possible. We hope to be able to complete the details of a different proof, in some future (including, possibly, the $C^{k}$-case). Since Hilbert's XIVth problem is solved negatively, the noncompact case is hopeless.

Now if $\xi$ is a smooth $G$-invariant vector field on $R^{n}$, one has in a natural way, a direct image of $\xi: \rho_{*} \xi$, which is a continuous vector field on the semialgebraic subset $\rho R^{n} \subset R^{p}$.

Proposition 3. If $\xi \in \Gamma^{\infty}\left(T R^{n}\right)^{G}$, then there is a smooth $\left(C^{\infty}\right)$ vector field $\eta \in \Gamma^{\infty}\left(T R^{p}\right)$ such that $\eta \mid \rho R^{n} \equiv \rho_{*} \xi$.

The same result is true for germs, and we deduce that if $\varphi(y) \in C^{\infty}(y)$, 
and $J(\varphi) \subset C^{\infty}(y)$ is the usual jacobian ideal of $\varphi$, then $\rho^{*} J(\varphi) \supset J_{G}\left(\rho^{*} \varphi\right)$. (Note that $\rho^{*} \varphi \in C^{\infty}(x)^{G}$.) This leads to one way of finding elements of finite codimension in $C^{\infty}(x)^{G}$. A better way is given by the following

Proposition 4. Let $f(x) \in C^{\infty}(x)^{G} \subset C^{\infty}(x)$ such that

$$
\operatorname{dim}_{R} C^{\infty}(x) / J(f)<\infty .
$$

Let $\varphi_{1}(x), \ldots, \varphi_{k}(x) \in C^{\infty}(x)$ be generators of $C^{\infty}(x) / J(f)$, as a vector space. Then $C^{\infty}(x)^{G} / J_{G}(f)$ is a finite dimensional vector space, generated by the averages of the $\varphi_{i}$ 's:

$$
\psi_{i}(x)=\int_{G} \varphi_{i}(g x) d \mu(g) \in C^{\infty}(x)^{G} .
$$

Here $d \mu(g)$ is the Haar measure of $G$. The general idea behind all this is that once one has a smooth version of Hilbert's finiteness theorem from the classical invariant theory, the Thom-Mather type theory of singularities can be extended to the case when a compact Lie group is operating. We plan to develop stability theory on these lines (see also [8]).

\section{BIBLIOGRAPHY}

1. E. Bierstone, Smooth functions invariant under the action of a finite group (to appear).

2. J. Dieudonné and J. Carrel, Invariant theory, old and new, Advances in Math. 4 (1970), 1-80. MR 41 \#186.

3. G. Lassalle, (to appear).

4. B. Malgrange, Ideals of differentiable functions, Studies in Math., no. 3, Tata Institute of Fundamental Research, Bombay; Oxford Univ. Press, London, 1967. MR 35 \#3446.

5. J. Mather, Right equivalence (to appear).

6. - Stability of $C^{\infty}$ mappings. I-IV, Ann. of Math. (2) 87 (1968), 89104; Ann. of Math. (2) 89 (1969), 254-291; Inst. Hautes Études Sci. Publ. Math. No. 35 (1968), 279-308; Inst. Hautes Études Sci. Publ. Math. No. 37 (1969), 223-248.

MR 38 \#726; 41 \#4582; 43 \#1215a, b.

7. V. Poénaru, Déploiement des fonctions G-invariantes (to appear).

8. - Un théorème des fonctions implicites . . . , Inst. Hautes Études Sci. Publ. Math. No. 38 (1970), 93-124.

9. G. Schwarz, Smooth functions invariant under the action of a compact Lie group, Topology 14 (1975), 63-69.

10. F. Sergeraert, Un théorème des fonctions implicites, Ann. Sci. École Norm. Sup. (1972), 559-660.

11. R. Thom, Stabilite structurelle et morphogenese, Benjamin, New York, 1972.

12. - La théorie des catastrophes, in Manifold, Warwick, 1973.

13. H. Weyl, The classical groups. Their invariants and representations, Princeton Univ. Press, Princeton, N. J., 1939. MR 1, 42.

14. V. M. Zakaljukin, $A$ theorem on versality, Funkcional. Anal. i Priložen. 7 (1973), no. 2, 28-31 = Functional Anal. Appl. 7 (1973), 110-112. MR 47 \#9670.

15. G. Glaeser, Fonctions composées différentiables, Ann. of Math. (2) 77 (1963), 193-209. MR $26 \# 624$. Sci., 1971.

16. R. Thom, Modèles mathématiques de la morphogenese, Inst. Hautes Études

DÉPARTMENT DE MATHÉMATIQUES, FACULTÉ DES SCIENCES D’ORSAY, 91 ORSAY, FRANCE 